\title{
Os entraves à população transgênero no acesso à saúde
}

\author{
Barriers to the transgender population in access to health
}

\author{
Barreras a la población transgénero en el acceso a la salud
}

\author{
Isabela Ferreira de Castro ${ }^{1 *}$, Alaôr Reis Filho1, Daniela Rezende Moreira1, Patrícia de Oliveira \\ Salgado', Silvia Almeida Cardoso'1.
}

\begin{abstract}
RESUMO
Objetivo: Compreender as barreiras enfrentadas pela população transgênero ao acessar o sistema de saúde, visto que esta questão é de grande importância em saúde coletiva e a literatura sobre o tema é escassa. Métodos: A pesquisa obteve uma amostra de oito artigos através de uma revisão de literatura, obtida na plataforma "Periódicos CAPES", no período entre 8 a 12 de abril de 2019, através da busca dos termos Transgender People e Health Service Need and Demand. Os critérios de seleção incluíam estudos publicados em inglês que abordavam as necessidades e barreiras dos pacientes transgênero no sistema de saúde, e a avaliação metodológica foi realizada com base nos itens do Strobe, Consort e Coreq. Resultados: Os resultados foram agrupados e discutidos em categorias. As principais dificuldades encontradas foram: desrespeito ao nome social, discriminação e a falta de capacitação dos profissionais. Considerações Finais: O despreparo da equipe de saúde, em diferentes cenários, contribui na construção das barreiras enfrentadas pela população trans, sendo primordial a implementação de políticas públicas e a qualificação profissional para atender as necessidades de saúde desse grupo.
\end{abstract}

Palavras-chave: Pessoas transgênero, Serviços de saúde para pessoas transgênero, Necessidades e demandas de serviços de saúde.

\section{ABSTRACT}

Objective: To understand the barriers faced by the transgender population when accessing the health system, since this issue is of great importance in public health and the literature on the subject is scarce. Methods: The research obtained a sample of eight articles through a literature review, obtained on the platform "Periódicos CAPES", in the period between April 8 to 12, 2019, through the search for the terms Transgender People and Health Service Need and Demand. The selection criteria included studies published in English that addressed the needs and barriers of transgender patients in the health system, and the methodological assessment was carried out based on items from Strobe, Consort and Coreq. Results: The results were grouped and discussed in categories. The main difficulties encountered were: disrespect to the social name, discrimination and the lack of training of professionals. Final Considerations: The unpreparedness of the health team, in different scenarios, contributes to the construction of the barriers faced by the trans population, being essential the implementation of public policies and professional qualification to meet the health needs of this group.

Keywords: Transgender persons, Health services for transgender persons, Health services needs and demand.

\section{RESUMEN}

Objetivo: Comprender las barreras que enfrenta la población transgénero al acceder al sistema de salud, ya que este tema es de gran importancia en la salud pública y la literatura sobre el tema es escasa. Métodos: La investigación obtuvo una muestra de ocho artículos a través de una revisión de literatura, obtenida en la plataforma "Periódicos CAPES", en el período comprendido entre el 8 y el 12 de abril de 2019, a través de la búsqueda de los términos: Transgender People e Health Service Need and Demand. Los criterios de selección incluyeron estudios publicados en inglés que abordaban las necesidades y barreras de los pacientes transgénero en el sistema de salud, y la evaluación metodológica se realizó con base en elementos de Strobe, Consort y Coreq. Resultados: Los resultados se agruparon y discutieron en categorías. Las principales dificultades encontradas fueron: falta de respeto al nombre social, discriminación y falta de formación de los profesionales. Consideraciones finales: La falta de preparación del equipo de salud, en diferentes escenarios, contribuye a la construcción de las barreras que enfrenta la población trans, siendo fundamental la implementación de políticas públicas y la calificación profesional para atender las necesidades de salud de este grupo.

Palabras clave: Personas transgénero, Servicios de salud para las personas transgénero, Necesidades y demandas de servicios de salud.

1 Universidade Federal de Viçosa, Viçosa - MG. *E-mail: isabelaferreiracastro@yahoo.com.br 


\section{INTRODUÇÃO}

Quando se trata da população transgênero, a literatura é escassa em todo o mundo. A carência por dados populacionais, demográficos, socioeconômicos e educacionais é visível e, quando presentes, não traz exatidão ou estão subestimados (EYSSEL J, et al., 2017).

No Brasil, o governo voltou sua atenção para a saúde dessa população no início da década de 1990 devido ao crescente número de casos de infecções pelo Vírus da Imunodeficiência Humana (HIV) no país, principalmente, entre as pessoas homossexuais. Em 1997, o Sistema Único de Saúde (SUS), implantou orientações para condutas destinadas à terapia para pessoas transgênero, com uso de hormônios esteróides, acompanhamento psicológico e procedimentos cirúrgicos (COSTA AB, et al., 2016).

Já o Processo Transexualizador no SUS, foi a partir da Portaria no $1.707 / G M / M S$, de 18 de agosto de 2008 e da Portaria no 457/SAS/MS, de 19 de agosto de 2008. Tais portarias estavam pautadas na habilitação de serviços em hospitais universitários e na realização de procedimentos hospitalares (MINISTÉRIO DA SAÚDE, 2019).

Apesar da carência literária sobre a saúde da população transgênero, entidades como a Organização Mundial da Saúde (OMS), a Transgender Europe (TGEU) e a Associação Nacional de Travestis e Transexuais (ANTRA), vêm apontando a importância e a necessidade de ampliar essa discussão, visto que apresenta maiores taxas de transtornos psiquiátricos que a população geral (WINTER S, 2009; HEYLENS G, et al., 2014; CHEUNG AS, et al., 2018; TRANSGENDER EUROPE, 2017).

Dados presentes no dossiê sobre assassinatos e violência contra a população trans e travestis, publicado pela ANTRA e pelo Instituto Brasileiro Trans de Educação (IBTE) em 2020, revelam que esses indivíduos encontram-se socialmente marginalizados, com menor acesso aos serviços essenciais como educação, saúde, incluindo mercado de trabalho formal e políticas públicas (BENEVIDES BG e NOGUEIRA SNB, 2020).

Essa ausência de direitos conjuga com outra informação presente no documento, a ocupação do Brasil em primeiro lugar no ranking mundial dos homicídios contra essa população. No entanto, não revelam de modo concludente os motivos dessas pessoas não integrarem os serviços primordiais, em especial de saúde, uma vez que apresentam maiores índices de distúrbios psicológicos e psiquiátricos.

Entendendo que o preconceito e a discriminação diariamente vividos por essa população, no Brasil, não devem ser os únicos fatores envolvidos com os dados alarmantes, e em virtude da importância desse tema na saúde coletiva e sua escassez na literatura, visualizamos que é necessário aprofundar o conhecimento sobre o acesso ao sistema de saúde dos indivíduos transgênero. Por esse motivo, a presente revisão tem como objetivo realizar um levantamento na literatura sobre as barreiras e as dificuldades enfrentadas por essa população quanto à questão de saúde e ao acesso a seus serviços.

\section{MÉTODOS}

O presente estudo consiste em uma revisão integrativa de literatura que seguiu cinco etapas: (i) identificação da questão de pesquisa; (ii) definição dos critérios de inclusão e exclusão de estudos; (iii) categorização e avaliação dos estudos; (iv) extração e interpretação dos resultados; e (v) síntese do conhecimento (MENDES K, et al., 2008). A questão norteadora deste estudo foi: Quais as barreiras e dificuldades enfrentadas pela população de transgênero quanto à saúde e o acesso a seus serviços?

Realizou-se a busca dos estudos na plataforma "Periódicos CAPES", no período dos dias 8 a 12 de abril de 2019, com os termos Transgender People e Health Service Need and Demand, seguidos dos refinamentos Humans, Medicine, Public health, Gender, Transgender people, Gender identity, Transgender e Humans rights.

Os critérios de seleção delimitados foram estudos primários que abordavam as necessidades e barreiras dos pacientes transgênero no sistema de saúde e publicados na língua inglesa. Foram excluídos os artigos que não abordavam, exclusivamente, a população transgênero. 
A avaliação da qualidade metodológica dos estudos foi realizada com base nos itens do Strobe (Strengthening the Reporting of Observational Studies in Epidemiology), do Coreq (Consolidated Criteria for Reporting Qualitative Research) e do Consort (Consolidated Standards of Reporting Trials) (MALTA M, et al., 2010; SCHULZ KF, et al., 2010; TONG A, et al., 2007).

Estes conjuntos de critérios foram publicados em 2010, 2007 e 2010, e elencam um checklist de recomendações para estudos observacionais, qualitativos e ensaios clínicos randomizados, respectivamente. Considerou-se ter adequada qualidade metodológica os artigos que apresentaram concordância com $50 \%$ ou mais dos itens dos instrumentos utilizados.

Utilizou-se um instrumento elaborado pelos autores para a extração e apresentação dos dados de interesse, contendo: título, objetivo, delineamento, casuística, discussão, resultados e conclusão no artigo. Os dados foram extraídos por dois avaliadores de forma independente. As inconsistências foram resolvidas por um terceiro avaliador (Figura 1).

Figura 1 - Fluxograma de informação sobre a identificação, triagem, elegibilidade e seleção de artigos para a revisão.

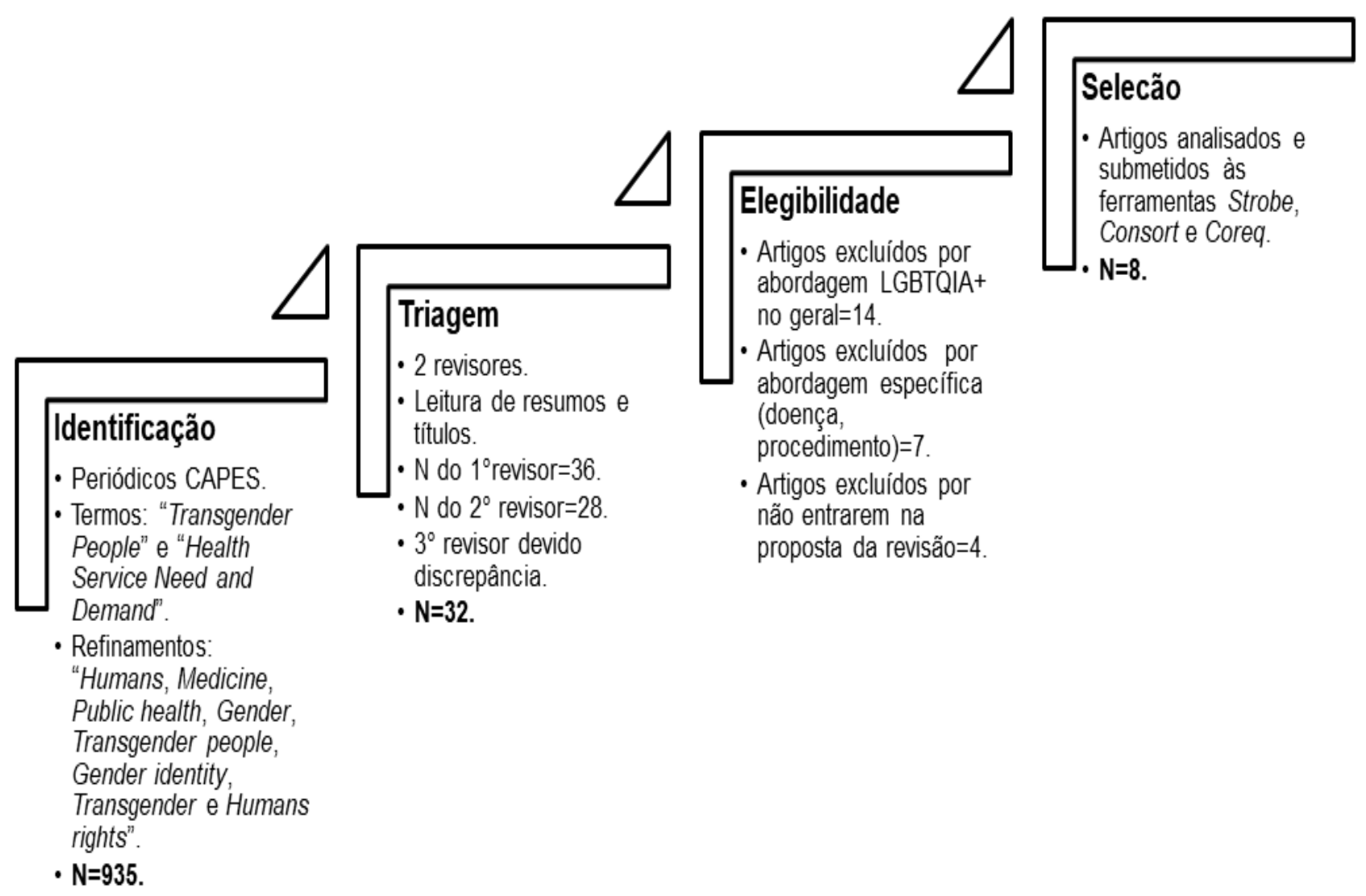

Fonte: Castro IF, et al., 2020.

\section{RESULTADOS}

Todos os oito estudos preencheram os critérios de elegibilidade, sendo quatro realizados nos Estados Unidos da América (EUA), dois no Brasil, um no Reino Unido e um na Alemanha. Dos artigos selecionados quatro eram estudos qualitativos, dois estudos transversais, um coorte, um quantitativo do tipo descritivo. Os trabalhos foram publicados entre 2008 e 2018 (Quadro 1).

Os oito artigos selecionados abordam sobre acesso e atendimento da população transgênero em relação às suas demandas de saúde. Cinco estudos descrevem as demandas em serviços especializados para saúde transgênero e os outros três utilizaram cenários diferentes para esta avaliação. 
Quadro 1 - Características dos artigos selecionados.

\begin{tabular}{|c|c|c|c|c|}
\hline $\begin{array}{c}\text { Artigos } \\
\text { Selecionados }\end{array}$ & $\begin{array}{l}\text { Desenho do } \\
\text { estudo }\end{array}$ & População e cenário de estudo & $\begin{array}{l}\text { Fonte de dados e } \\
\text { métodos utilizados }\end{array}$ & Objetivo do estudo \\
\hline $\begin{array}{l}\text { DEWEY JM, } \\
\text { 2008. }\end{array}$ & Qualitativo & $\begin{array}{l}\text { Pessoas transgênero de uma } \\
\text { cidade do centro oeste EUA. }\end{array}$ & $\begin{array}{l}\text { Coleta de dados } \\
\text { primários (Entrevistas). }\end{array}$ & $\begin{array}{c}\text { Examinar relatos de pacientes transgênero e seu envolvimento com } \\
\text { profissionais médicos. }\end{array}$ \\
\hline $\begin{array}{l}\text { REISNER SL, et } \\
\text { al., } 2013 .\end{array}$ & $\begin{array}{l}\text { Quantitativo } \\
\text { e qualitativo }\end{array}$ & Pessoas Transmasculinas. & $\begin{array}{l}\text { Questionário e oficina } \\
\text { de saúde com } \\
\text { perguntas discursivas. }\end{array}$ & $\begin{array}{l}\text { Investigar formativamente a saúde e as necessidades de saúde } \\
\text { percebidas de adultos transmasculinos. }\end{array}$ \\
\hline $\begin{array}{l}\text { XAVIER J, et al., } \\
2013 .\end{array}$ & Qualitativo & $\begin{array}{l}\text { Pessoas transgênero na } \\
\text { Virgínia. }\end{array}$ & Grupos focais. & $\begin{array}{l}\text { Identificar os fatores associados ao maior risco de infecção pelo HIV } \\
\text { e os principais determinantes sociais do estado de saúde entre } \\
\text { pessoas trans na Virgínia. Secundariamente, examinamos as } \\
\text { maneiras pelas quais as pessoas transgênero acessam atualmente } \\
\text { serviços médicos, de saúde mental, relacionados a transgênero e } \\
\text { HIV na Virgínia. }\end{array}$ \\
\hline $\begin{array}{l}\text { DAVIES A, et } \\
\text { al., } 2014 .\end{array}$ & Quantitativo & $\begin{array}{l}\text { Pessoas transgênero em uma } \\
\text { clínica de identidade de gênero } \\
\text { no Reino unido. }\end{array}$ & Questionário. & $\begin{array}{l}\text { Entender melhor os níveis de satisfação com a oferta atual de } \\
\text { serviços de clínica de identidade de gênero (GICs) e identificar } \\
\text { áreas para melhoria. }\end{array}$ \\
\hline $\begin{array}{l}\text { TAGLIAMENTO } \\
\text { G e PAIVA V } \\
2016 .\end{array}$ & Qualitativo & $\begin{array}{l}\text { Pessoas transgênero em uma } \\
\text { ONG e em um serviço de saúde } \\
\text { especializado. }\end{array}$ & $\begin{array}{l}\text { Entrevistas e estudo } \\
\text { direto de observação. }\end{array}$ & $\begin{array}{l}\text { Compreender o acesso de pessoas trans ao sistema público de } \\
\text { saúde brasileiro. }\end{array}$ \\
\hline $\begin{array}{l}\text { DIETERT M, et } \\
\text { al., } 2017 .\end{array}$ & $\begin{array}{l}\text { Qualitativo e } \\
\text { quantitativo }\end{array}$ & $\begin{array}{l}\text { Veteranos militares transgênero. } \\
\text { Serviços de saúde prestados } \\
\text { pela Administração de Saúde } \\
\text { dos Veteranos (VHA). }\end{array}$ & $\begin{array}{l}\text { Entrevista e } \\
\text { questionário. }\end{array}$ & $\begin{array}{l}\text { Investigar as experiências de uma amostra de veteranos } \\
\text { transgêneros em relação às suas experiências com os serviços de } \\
\text { saúde prestados pelo VHA. }\end{array}$ \\
\hline $\begin{array}{l}\text { EYSSEL J, et } \\
\text { al., } 2017 .\end{array}$ & Quantitativo & $\begin{array}{l}\text { Pessoas transgênero. } \\
\text { Centro interdisciplinar sobre } \\
\text { cuidados da saúde trans na } \\
\text { Alemanha. }\end{array}$ & Questionário. & $\begin{array}{l}\text { Investigar as necessidades e preocupações que indivíduos } \\
\text { transgênero têm com relação à saúde trans em centros } \\
\text { interdisciplinares para pessoas transgênero. }\end{array}$ \\
\hline $\begin{array}{l}\text { COSTA AB, et } \\
\text { al., } 2016 .\end{array}$ & Quantitativo & $\begin{array}{c}\text { Pessoas transgênero. } \\
\text { Programas de identidade de } \\
\text { gênero no Rio Grande do Sul e } \\
\text { São Paulo. }\end{array}$ & Questionário. & $\begin{array}{c}\text { Avaliar as necessidades de saúde de pessoas transgênero e as } \\
\text { barreiras de acesso em dois estados brasileiros. }\end{array}$ \\
\hline
\end{tabular}

Fonte: CASTRO IF, et al., 2020. 
Para melhor compreensão, os artigos que compuseram a amostra deste estudo foram categorizados em atendimento em serviço de saúde, discriminação no sistema de saúde, capacitação dos profissionais de saúde, hormonização e modificação corporal e serão abordados a seguir.

\section{Atendimento em serviço de saúde}

Em relação ao desconforto das pessoas transgênero em expressar suas necessidades para os profissionais de saúde, um estudo brasileiro com participantes de programa de identidade de gênero identificou que, no grupo amostral, $148(25,6 \%)$ relatam sentir-se muito desconfortáveis, 211 (36,5\%) desconfortáveis, $149(25,8 \%)$ confortáveis e apenas $70(12,1 \%)$ afirmaram sentir-se muito confortáveis durante o atendimento (COSTA AB, et al., 2016). Reisner SL, et al. (2013) já haviam demonstrado anteriormente que, entre os entrevistados transmasculinos adultos, 48,7\% relatam ter evitado ou atrasado 0 atendimento de saúde nos últimos 12 meses por seu status de transgênero ou identidade de gênero. De forma similar, Dietert M, et al. (2017) apontam que alguns entrevistados, por serem transgênero, não se sentiram confortáveis em procurar atendimento, e que a assistência integral foi inadequada.

Costa $A B$, et al. (2016) reforçam, ainda, que a maioria da população transgênero pontua a necessidade de apresentar e ensinar aos profissionais de saúde suas necessidades, sendo constatado em 376 (62,7\%) participantes.

\section{Discriminação no sistema de saúde}

Costa $A B$, et al. (2016) encontraram dados alarmantes: 270 (58,7\%) dos transgênero relataram terem sidos discriminados durante o atendimento e $249(43,2 \%)$ disseram evitar o serviço de saúde por já terem sofrido discriminação em atendimentos anteriores.

Entre as formas de discriminação descritas nesse estudo, a mais prevalente foi desrespeito ao nome social, seguida por profissionais que desencorajam a explorar seu gênero, que utilizam linguagem ofensiva, que ridicularizam sua condição trans, que se negaram a resolver ou discutir seus problemas, que afirmam aos pacientes não serem transgênero, ter atendimento interrompido ou recusado e, por último, profissionais que recusaram a examinar alguma parte do seu corpo.

De forma similar, Tagliamento $G$ e Paiva $V(2016)$ pesquisaram pessoas transgênero de serviços especializados ou organizações não governamentais, e descreveram diferentes formas de discriminação sofrida dentro do sistema de saúde nacional, como barreiras que surgiram imediatamente após a chegada ao serviço, quando os atendentes solicitavam os documentos para cadastro inicial.

Xavier J, et al. (2013) já haviam descrito, nos EUA, problemas com documentos de identidade e com o nome social. Muitos mencionaram hostilidade, insensibilidade, desrespeito e franca discriminação por parte dos prestadores de serviços médicos.

O medo de uma reação hostil ou insensível levou alguns participantes a não revelarem seu status de transgênero ao profissional com quem não tinham muito contato. Reisner SL, et al. (2013) corroboram com esses dados ao descrever que, quase metade dos pacientes transmasculinos adultos, relataram discriminação por algum médico durante a vida.

\section{Capacitação dos profissionais de saúde}

Dewey JM (2008) descreveu que alguns indivíduos transgênero relatam sentir desconforto durante o atendimento médico e, de forma mais impactante relatou, ainda, a recusa em atendê-los. Nesse estudo, realizado nos EUA, o autor demonstrou que a comunidade transgênero tem mais conhecimento sobre 0 processo transexualizador que as equipes médicas.

Costa $A B$, et al. (2016), quanto à formação dos profissionais de saúde, relatam que $24,6 \%$ desses trabalhadores afirmaram não saber sobre os cuidados em relação à saúde de pessoas transgênero. Resultado semelhante encontrado por Tagliamento G e Paiva V (2016), no qual todos os entrevistados apontaram a inadequada formação dos profissionais em atendê-los. Dietert M, et al. (2017), no serviço de saúde à veteranos norte-americanos, evidenciaram falta de treinamento da equipe para a questão de saúde relacionadas à população transgênero. 
Não obstante, Eyssel J, et al. (2017) relatam que as principais razões para o atendimento de pessoas transgênero em centro interdisciplinar de cuidados com a saúde transgênero na Alemanha são a provisão de um 'espaço seguro' ( $n=228)$, a formação profissional $(n=132)$, o tratamento holístico $(n=35)$ e os aspectos organizacionais.

Entre os aspectos negativos nesse ambiente, têm-se: a segregação de pessoas transgênero do sistema de saúde geral $(n=6)$, as limitações da livre escolha de profissionais por pacientes $(n=3)$ e a baixa acessibilidade ao centro $(n=3)$.

Xavier J, et al. (2013) descreveram que durante o atendimento em serviço não especializado, após a identificação da transexualidade, alguns médicos se concentraram em seu status de transgênero e seus problemas médicos imediatos passaram a ser secundários.

Nesse estudo, a deficiência na formação e capacitação dos médicos ficou mais evidente no atendimento ginecológico, uma necessidade contínua da maioria dos transgênero masculinos, que quando conseguiam atendimento, relataram que eram emocional e fisicamente dolorosos devido à insensibilidade profissional. Muitos desses participantes simplesmente optaram por renunciar ao cuidado ginecológico.

\section{Hormonização}

O acesso à terapia hormonal é uma questão muito relevante para a população transgênero (DIETERT M, et al., 2017). Os autores relatam que muitos procuram o serviço de saúde com informações prévias sobre o processo transexualizador e que a hormonização é uma prioridade para a maioria.

Em estudo brasileiro, realizado com participantes transgênero de programa de identidade de gênero, $83,9 \%$ dos transgênero homens e $48,8 \%$ das transgênero mulheres afirmaram fazer uso de hormônios. Dentre os que afirmaram nunca ter feito uso de hormônios, $34,2 \%$ não decidiram se hormonização é adequada para seu caso, 42,2\% não encontraram médico para fazer a prescrição, 13,1\% não pretendem usar hormônios e $6,8 \%$ estavam prestes a começar (COSTA AB, et al., 2016).

As barreiras no acesso hormonal são a falha dos planos de saúde para cobrir esses serviços, a impossibilidade de arcar com os custos e a escassez de profissionais dispostos a prescrever (XAVIER J, et al., 2013). Um estudo conduzido por Davies A, et al. (2013), em clínica de identidade de gênero no Reino Unido, apresentou que $61 \%$ dos indivíduos transgênero estavam satisfeitos ou muito satisfeitos com as informações fornecidas pelos médicos sobre o tratamento hormonal, reforçando a necessidade da capacitação profissional em relação às demandas específicas da população transgênero.

\section{Modificação corporal}

A hormonização e a cirurgia de resignação sexual são importantes agentes afirmadores do gênero (REISNER SL, et al., 2013). Costa AB, et al. (2016) relatam que 23,9\% dos indivíduos transgênero masculinos e femininos afirmaram já ter feito algum procedimento de modificação corporal.

A maioria realizou em clínicas ou hospitais privados, seguidos de serviços clandestinos e um número menor em locais públicos em outro país. Dos que afirmaram não ter realizado nenhum procedimento, $64,2 \%$ asseguraram não ter feito por falta de recurso financeiro, 17,5\% por medo, $15,2 \%$ por não querer se autoaplicar, $13,8 \%$ por não querer ser atendido em serviço público, 12,9\% tentaram sem sucesso, 7,1\% estavam em processo e 1,1\%, em dúvida. Xavier J, et al. (2013) identificaram o uso de silicone industrial como alternativa à hormonização, o que também foi descrito nas mulheres transgênero na busca de resultados mais rápido.

\section{DISCUSSÃO}

Um importante entrave encontrado na avaliação do acesso da população transgênero ao serviço de saúde, nesta revisão, foi o recrutamento de artigos científicos que analisassem a questão transgênero puramente sem associá-la ao HIV, situação também encontrada em uma metanálise sobre a prevalência do transexualidade (ARCELUS J, et al., 2015). Os autores demonstraram que o aumento dessa prevalência, nos últimos 50 anos, juntamente com dificuldades de acesso aos sistemas de saúde, reforça a importância de ampliar a discussão sobre essa temática. 
Os estudos em diferentes países e culturas demonstram que a população transgênero enfrenta problemas comuns em relação ao acesso aos sistemas de saúde. Entre as principais barreiras descritas e encontradas estão: a falta de respeito ao nome social; o despreparo da equipe médica e demais profissionais de saúde sobre a temática transgênero; a falta de confiança no profissional, fazendo com que muitos não procurem atendimento; além de relatos frequentes das mais diversas formas de discriminação.

Em sua revisão integrativa, Rocon PC, et al. (2020) buscaram avaliar o acesso a saúde nacional da população transgênero após o ano de 2008, quando foi criado o processo transexualizador do SUS, e a discriminação nesses ambientes também se apresentou como uma importante barreira ao acesso.

Ramos LS, et al. (2020) em sua revisão narrativa sobre a humanização da atenção básica a saúde de pessoas travestis e transexuais, também identificou a discriminação, a falta de preparo dos profissionais de saúde e os preconceitos, dificultando o atendimento humanizado a esta população e reforçando, assim, necessidade de constante capacitação dos profissionais juntamente com a implantação de políticas públicas.

Nossos resultados demonstraram que a discriminação ocorre com frequência durante o atendimento e que ela passa a ser um dos impedimentos ao acesso ao serviço de saúde para pessoas transgênero, não só para os que buscam atendimento para o processo transexualizador, mas para as mais diversas necessidades de saúde, incluindo tratamento antirretroviral e educação sexual.

Um estudo, realizado nos EUA por Xavier J, et al. (2013), demonstrou que a taxa de infecção por HIV entre transgênero mulheres é alto e, tendo como principais razões, os comportamentos sexuais de risco, o compartilhamento de agulhas durante a injeção de hormônios ou uso de drogas.

Práticas essas que poderiam ser mitigadas com orientação técnica adequada. Verifica-se que a falta de sensibilidade por parte dos profissionais de saúde quanto ao desrespeito à identidade de gênero, influencia adversamente as pessoas transgênero e as afasta do acesso a tratamentos e serviços a de saúde (LOMBARDI E, 2001).

Eyssel J, et al. (2017) apresentaram que os centros especializados no atendimento transgênero tem vantagens, como profissionais capacitados, contudo apontou pontos negativos, como a segregação no atendimento e a centralização, dificultando o acesso.

Ademais, são poucos os centros especialistas em atendimento a pessoas transgênero, além disso, aqueles existentes estão em locais de difícil acesso, não centrais, o que leva a comportamentos de risco como, compra de hormônio sem prescrição e acompanhamento médico, compartilhamento de agulha para sua administração, uso de silicone industrial para conseguir realizar sua transição (LOMBARDI E, 2001).

A necessidade de profissionais de saúde atentos às questões de gênero e demandas da população transgênero, assim como a permanente capacitação, ficam evidentes quando se descreve a recusa de atendimento pela falta de conhecimento, ou mesmo quando realizado de forma inadequada seja em questões gerais de saúde ou específicas ao público.

Um estudo realizado por Almeida JSM, et al. (2018), em um hospital universitário brasileiro especializado no processo transexualizador, exemplifica a carência na formação profissional em relação ao gênero, visto que os residentes em enfermagem relataram não ter tido formação adequada sobre a temática.

Além da discriminação durante o atendimento e do despreparo desses profissionais para as demandas de pacientes transgênero, foi possível identificar a dificuldade ao acesso a hormonização e as modificações corporais, que são muitas vezes necessárias e procuradas para a afirmação do gênero.

Uma das principais demandas de saúde específicas da população transgênero é a hormonização. Esta revisão observou que os pacientes encontram dificuldade não só pela falta de capacitação médica na prescrição adequada de hormônios, ou mesmo que estejam dispostos a isto, assim como a sua aquisição e a falta de cobertura pelos planos de saúde.

Esses problemas levam ao uso indiscriminado e sem o devido acompanhamento médico, resultam em complicações de saúde. Lee H, et al. (2018), na Coreia, objetivaram conhecer a experiência e as barreiras no processo transexualizador. 
Em relação à hormonização, os autores encontraram que $81,5 \%$ estavam em uso, os que não estavam foram questionados quanto ao motivo e a principal resposta foi falta de recurso financeiro para comprar; $25,1 \%$ já haviam comprado sem prescrição, desse grupo, 55,7\% disseram ter feito isso por não ter tido o diagnóstico psiquiátrico (LEE H, et al., 2018). Os resultados obtidos por esse estudo coreano vão de encontro aos desta revisão ao afirmar que a maior parte das pessoas transgênero usa hormônio e entre os que não usam, os motivos se assemelham: falta de recurso financeiro e de médicos capacitados para prescrever.

Hembree WC, et al. (2017), em sua diretriz prática para tratamento endocrinológico de pessoas disfóricas/incongruentes de gênero, sugerem avaliação clínica regular para mudanças físicas e potenciais eventos adversos em resposta aos hormônios esteroides sexuais, além de monitoramento laboratorial dos níveis hormonais a cada três meses durante o primeiro ano de terapia hormonal para homens e mulheres transexuais e, após, uma ou duas vezes por ano.

Essa questão elucida uma preocupação quando apontam a dificuldade em encontrar médicos dispostos ou que saibam conduzir o tratamento. Assim, sem acesso à prescrição adequada e seu acompanhamento, os pacientes ficam expostos a diversas complicações e problemas de saúde quando fazem o uso indiscriminado dos hormônios, colocando em risco a própria vida.

Procedimentos de modificação corporal são uma importante forma de afirmação do gênero. A dificuldade de acesso a eles acarreta na busca por clínicas clandestinas e no uso indiscriminado de substâncias não permitidas, que muitas vezes são autoaplicadas, e prejudiciais à saúde. Aguayo-Romero RA, et al.

(2015) pontuaram que esses procedimentos, como uso de silicone injetável ou próteses, são considerados tratamentos estéticos e não são cobertos pelo serviço de saúde da Colômbia e em muitos países, levando, assim, à autoadministração de silicone de grau industrial e sem conhecimento dos possíveis efeitos adversos. No Brasil, alguns serviços do SUS oferecem os procedimentos de modificação corporal sem custo, mas o acesso é restrito, localizados em poucas cidades, geralmente nos grandes centros, além de oferecerem com poucas vagas para atendimento.

\section{CONSIDERAÇÕES FINAIS}

Apesar de a população transgênero estar ganhando visibilidade, foi possível perceber que, mesmo em cenários diferentes, essa população enfrenta as mesmas dificuldades ao buscar acesso à saúde. Entre as principais dificuldades se destacam a discriminação, o não respeito ao nome social, a falta de capacitação dos profissionais de saúde para as necessidades desse público, o uso de hormonização e procedimentos de modificações corporais sem acompanhamento adequado. Isso reforça a importância da discussão de gênero na formação dos profissionais de saúde. Também se faz necessário o desenvolvimento de pesquisa que produzam dados necessários para auxiliar na tomada de decisão sobre a saúde específica dos transgênero melhorando, assim, seu atendimento de forma ética, humana e com respeito. Como limitação do estudo, encontra-se o fato de a maior parte dos artigos sobre pessoas transgênero estar associada à infecção pelo HIV, bem como a carência de estudos nessa área específica. Contudo, acredita-se que, com aumento da procura desta população pelos serviços de saúde, esta revisão tem sua contribuição ao mostrar que é necessário discutir e abordar mais a temática transgênero na formação dos profissionais de saúde preparando-os, assim, melhor para o atendimento.

\section{REFERÊNCIAS}

1. AGUAYO-ROMERO RA, et al. Gender Affirmation and Body Modification Among Transgender Persons in Bogotá, Colombia. International Journal of Transgenderism, 2015; 16(2): 103-115.

2. ALMEIDA JSM, et al. Cuidar de pessoas transexuais na ótica dos residentes de enfermagem. Revista enfermagem UERJ, 2018; 26: e32030.

3. ARCELUS J, et al. Systematic review and meta-analysis of prevalence studies in transsexualism. European Psychiatry, 2015; 30(1): 807-815.

4. BENEVIDES BG, NOGUEIRA SNB. Dossiê dos assassinatos e da violência contra travestis e transexuais brasileiras em 2019. São Paulo: Expressão Popular, ANTRA, IBTE, 2020; 80p.

5. CHEUNG AS, et al. Sociodemographic and clinical characteristics of transgender adults in Australia. Transgender Health, 2018; 3(1): 229-238. 
6. COSTA AB, et al. Healthcare needs of and access barriers for brazilian transgender and gender diverse people. Journal of Immigrant and Minority Health, 2016; 20(1): 115-123.

7. DAVIES A, et al. Patient satisfaction with gender identity clinic services in the United Kingdom. Sexual and Relationship Therapy, 2013; 28(4): 400-418.

8. DEWEY JM. Knowledge Legitimacy: How trans-patient behavior supports and challenges current medical knowledge. Qualitative Health Research, 2008; 18(10): 1345-1355.

9. DIETERT M, et al. Addressing the needs of transgender military veterans: better access and more comprehensive care. Transgender Health, 2017; 2.1(1): 35-44.

10. EYSSEL J, et al. Needs and concerns of transgender individuals regarding interdisciplinar transgender healthcare: a non-clinical online survey. PLoS ONE, 2017; 12(8): e0183014.

11. HEMBREE WC, et al. Endocrine Treatment of Gender-Dysphoric/Gender-Incongruent Persons: An Endocrine Society Clinical Practice Guideline. The Journal of Clinical Endocrinology \& Metabolism, 2017; 102(11): 3869-3903.

12. HEYLENS G, et al. Psychiatric characteristics in transsexual individuals: multicentre study in four european countries. The British Journal of Psychiatry, 2014; 204(2): 151-156.

13. LEE $\mathrm{H}$, et al. Experiences of and barriers to transition-related healthcare among Korean transgender adults: focus on gender identity disorder diagnosis, hormone therapy, and sex reassignment surgery. Epidemiology and Health, 2018; 40: e2018005.

14. LOMBARDI E. Enhancing Transgender Health Care. American Journal of Public Health, 2001; 91(6): 869-872.

15. MALTA M, et al. Iniciativa STROBE: subsídios para a comunicação de estudos observacionais. Revista de Saúde Pública, 2010; 44(3): 559-65.

16. MENDES K, et al. Revisão integrativa: método de pesquisa para a incorporação de evidências na saúde e na enfermagem. Texto \& Contexto - Enfermagem, 2008; 17(4): 758-64.

17. MINISTERIO DA SAÚDE. Processo Transexualizador no SUS. 2018. Disponível em: http://www.saude.gov.br/atencao-especializada-e-hospitalar/especialidades/processo-transexualizador-no-sus. Acesso em: 12 jun. 2019.

18. RAMOS LS, et al. A humanização da atenção básica a saúde brasileira no atendimento de travestis e transexuais: uma revisão narrativa. Electronic Journal Collection Health, 2020; 44: e2770.

19. REISNER SL, et al. Female-to-male transmasculine adult health: a mixed-methods community-based needs assessment. Journal of the American Psychiatric Nurses Association, 2013; 19(5): 293-303.

20. ROCON PC, et al. Acesso à saúde pela população trans no Brasil: nas entrelinhas da revisão integrativa. Trabalho, Educação e Saúde, 2020; 18(1): e0023469.

21. SCHULZ KF, et al. CONSORT 2010 Statement: updated guidelines for reporting parallel group randomised trials. BMJ, 2010; 340: c332.

22. TAGLIAMENTO G, PAIVA V. Trans-specific health care: challenges in the context of new policies for transgender people. Journal of Homosexuality, 2016; 63(11): 1556-1572.

23. TONG A, et al. Consolidated criteria for reporting qualitative research (COREQ): a 32 -item checklist for interviews and focus groups. International Journal for Quality in Health Care, 2007; 19(6): 349-357.

24. TRANSGENDER EUROPE. World Mental Health Day: Transgender Europe publishes worrying results about trans people's mental health. 2017. Disponível em: https://tgeu.org/healthsurvey_release/. Acesso em: 6 maio 2019.

25. WINTER S. Cultural considerations for the World Professional Association for transgender health's standards of care: The Asian perspective. International Journal of Transgenderism, 2009; 11(1): 19-41.

26. XAVIER J, et al. Transgender health care access in Virginia: A qualitative study. International Journal of Transgenderism, 2013; 14(1): 3-17. 